\title{
THE MAXIMUM PRINCIPLE OF PONTRYAGIN IN CONTROL OF TWO- LEGGED ROBOT BASED ON HUMAN WALKING SYSTEM
}

\author{
K.K. ŻUR \\ Department of Production Management \\ Faculty of Management \\ Technical University of Bialystok \\ Ojca Tarasiuka 2 Street, 16-001 Kleosin, POLAND \\ E-mail: k.zur@pb.edu.pl
}

\begin{abstract}
In the paper a hypothesis about state equations of human gait is presented. Instantaneous normalized power developed by human muscles at particular joints of a leg is a control vector in state equations of the human walking system. The maximum principle of Pontryagin in analysis of dynamic human knee joint was presented. The discrete Hamilton function of a knee joint is similar to a discrete square function of normalized power developed by muscles at the knee joint. The results satisfy optimal conditions and could be applied in control of exoskeleton and DAR type robot.
\end{abstract}

Key words: state equations, maximum principle, optimal conditions, discrete Hamilton function.

\section{Introduction}

Many methods of scientific research on two-legged human locomotion have been presented in works (Bocardi and Pedotti, 1981; Winter, 1991; Jaworek, 1992; Żur and Jaworek, 2011; Żur, 2013). A great number measurements of human gait in the sagittal plane of motion for young as well as old people is presented in works (Winter, 1991; Jaworek, 1992). As a result of the long-term research a certain measure has been found by Jaworek (1992). The measure is called an assessment indicator and it has the following form

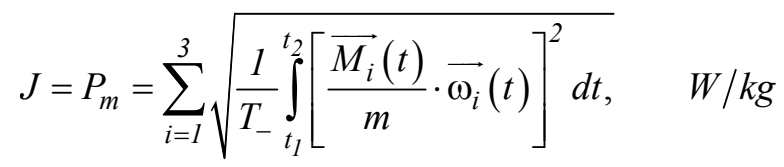

where: $P_{m}$ - an indicator of standardized power $[\mathrm{W} / \mathrm{kg}], \overrightarrow{\omega_{i}}(t)$ - the vector of angular velocity that is developed in the $i$-th axis of the joint $[\mathrm{rad} / \mathrm{s}], \frac{\overrightarrow{M_{i}}(t)}{m}$ - the normalized resultant vector of moment of the muscle forces, developed in the $i$-th joint $[N \circ \mathrm{m} / \mathrm{kg}], \mathrm{m}$ - the mass of human $[\mathrm{kg}], T_{-}=t_{2}-t_{1}$ - duration of support phase $[s]$.

The resultant vector of moment of muscle forces $\overrightarrow{M_{i}}(t)$ of the rotation axis of the $i$-th joint of human leg is the sum of three vectors

$$
\overrightarrow{M_{i}}=\overrightarrow{M_{i j}}+\overrightarrow{M_{i g}}+\overrightarrow{M_{i r}}
$$


where: $\overrightarrow{M_{i j}}$ - the vector of moment in the $i$-th joint developed by the inertia forces of the lower limb below the $i$-th joint $[N \circ m] \cdot \overrightarrow{M_{i g}}$ - the vector of moment in the $i$-th joint developed by the forces of gravity of the lower limb below the $i$-th joint $[N \circ m] . \overrightarrow{M_{i r}}$ - the vector of moment in the $i$-th joint developed by the reaction force of the ground $[N \circ m]$.

When the vector of moment $\overrightarrow{M_{i}}$ of formula (1) is changed into the vector of moment $\overrightarrow{M_{i r}}$ then an average power (developed by the leg muscles in support phase of a human foot during one cycle of motion) is obtained (Bocardi and Pedotti, 1981).

The functional (1.1) physically means an average quantity of power developed by the joint of the tested person in the support phase during movement on a flat surface.

In the paper the modification of the indicator $J$ to form $J_{i}^{*}$ was presented as follows

$$
J_{i}^{*}=\frac{1}{T_{-/+}} \sqrt{\int_{0}^{T_{-I+}} \frac{p_{i}^{2}(t)}{m^{2}} d t}, \quad W / k g
$$

where: $T_{-/+}$- the duration of one step that includes both support and swing phases of the lower leg $[s]$, $p_{i}(t) / m$ - a normalized instantaneous power developed by the muscle groups which operate the $i$-th joint $[\mathrm{W} / \mathrm{kg}], \mathrm{m}$ - mass of human $[\mathrm{kg}], i$ - an indicator that includes three main joints of the human leg (hip, knee and ankle joints).

\section{The maximum principle of Pontryagin in analysis of two-legged human gait}

In the work (Żur and Jaworek, 2011) a hypothesis about energy saving during human motion was presented as follows:

„typical human locomotion in the Earth's gravitational field minimizes the value of the functional $J_{i}^{*}(1.3)$ ”. In conclusion, the smaller the value of the functional, the better neuromuscular coordination during human locomotion. The consumption of energy of motion gets smaller during walking.

\subsection{State equations of human joints}

Żur and Jaworek $(2011 ; 2013)$ have suggested a new description of human locomotion by the method of space state. The hypothesis about the control vector $\underline{u}(t)$ in the walking system is as follows: "the control $\underline{u_{i}}(t)$ is the normalized power in the $i$-th main joint of a human leg during one single step". It follows that the control $\underline{u_{i}}(t)=p_{i}(t) / m$ has the following form

$$
\underline{u_{i}}(t)=\frac{p_{i}(t)}{m}=\frac{\overrightarrow{M_{i}}(t)}{m} \cdot \overrightarrow{\omega_{i}}(t), \quad W / k g
$$

where: $\overrightarrow{M_{i}}(t) / m$ - the normalized vector of moment developed in the $i$-th main joint $[N \circ \mathrm{m} / \mathrm{kg}], \overrightarrow{\omega_{i}}(t)-$ the vector of angular velocity developed in the $i$-th axis of human joint $[\mathrm{rad} / \mathrm{s}], \mathrm{m}$ - mass of the human $[\mathrm{kg}]$.

In order to describe the process of human movement in the space state, three relative angles are established (Fig.1) as the coordinates of the state, as follows: $\phi_{B}$ - the angle between the torso and thigh 
$[\mathrm{rad}], \phi_{K}$ - the angle between the thigh and shin $[\mathrm{rad}], \phi_{S G}$ - the angle between the shin and foot $[\mathrm{rad}]$. According to the hypothesis about the control vector $\underline{u_{i}}(t)$, the equation of state has the following form

$$
\underline{\dot{\phi}_{i}}(t)=\underline{a_{i}}(t) \cdot \underline{\phi_{i}}(t)+\underline{b_{i}}(t) \cdot \underline{p_{i}}(t) / m
$$

with the initial condition

$$
\underline{\phi_{i}}\left(t=t_{0}\right)=\underline{\phi_{i 0}}=C O N S T
$$

where: $\phi_{i}(t)$ - the relative angle between the main elements of human leg, i.e., the torso and thigh, the thigh and shin, the shin and foot $[\mathrm{rad}], \underline{\dot{\phi}_{i}}(t)$ - the angular velocity developed in the $i$-th joint of a human leg, i.e., of the hip, knee and ankle joints $[\mathrm{rad} / \mathrm{s}], \underline{p_{i}}(t) / m$ - the normalized power developed by the muscle groups which operate the hip, knee and ankle joints $[\mathrm{W} / \mathrm{kg}], a_{i}(t)$ and $b_{i}(t)$ - the coefficients of adequate state matrix $[\mathrm{A}]$ in $[1 / \mathrm{s}]$ and control matrix $[\mathrm{B}]$ in $[\mathrm{rad} \cdot \mathrm{kg} / \mathrm{J}]$.

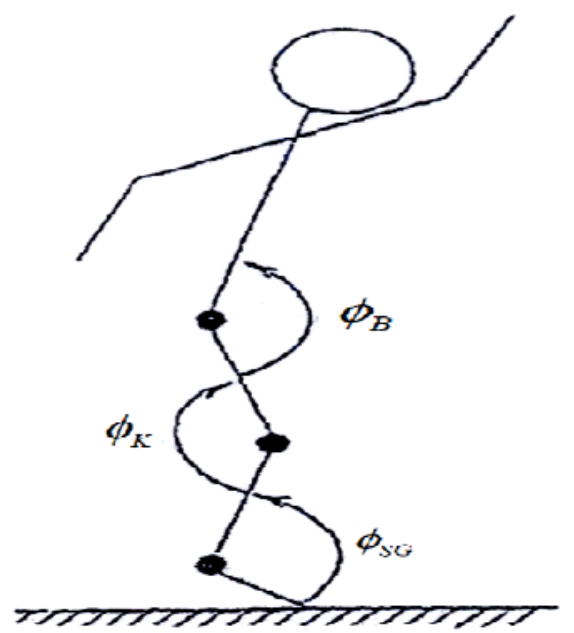

Fig.1. The diagram of human (robot) walking system with the coordinates of the state, where: $\phi_{B}-$ the angle between the torso and thigh, $\phi_{K}$ - the angle between the thigh and shin, $\phi_{S G}$ - the angle between the shin and foot.

A description of the human walking system in the state space allows a reduction of the number of differential equations of 60 degrees of freedom for the lower human limb to three state equations that describe three main joints of a human leg, i.e., the hip, knee and ankle joints. Winter in the work (1991) described kinematic and dynamic data for a group of young, healthy and physically fit people. He also placed there discrete values of normalized power $p_{i}(t) / m$ (Fig.2) developed by the main muscle groups and discrete values of relative angles $\phi_{i}(t)$ (Fig.3) respectively for the hip, knee and ankle joints for one single step. 


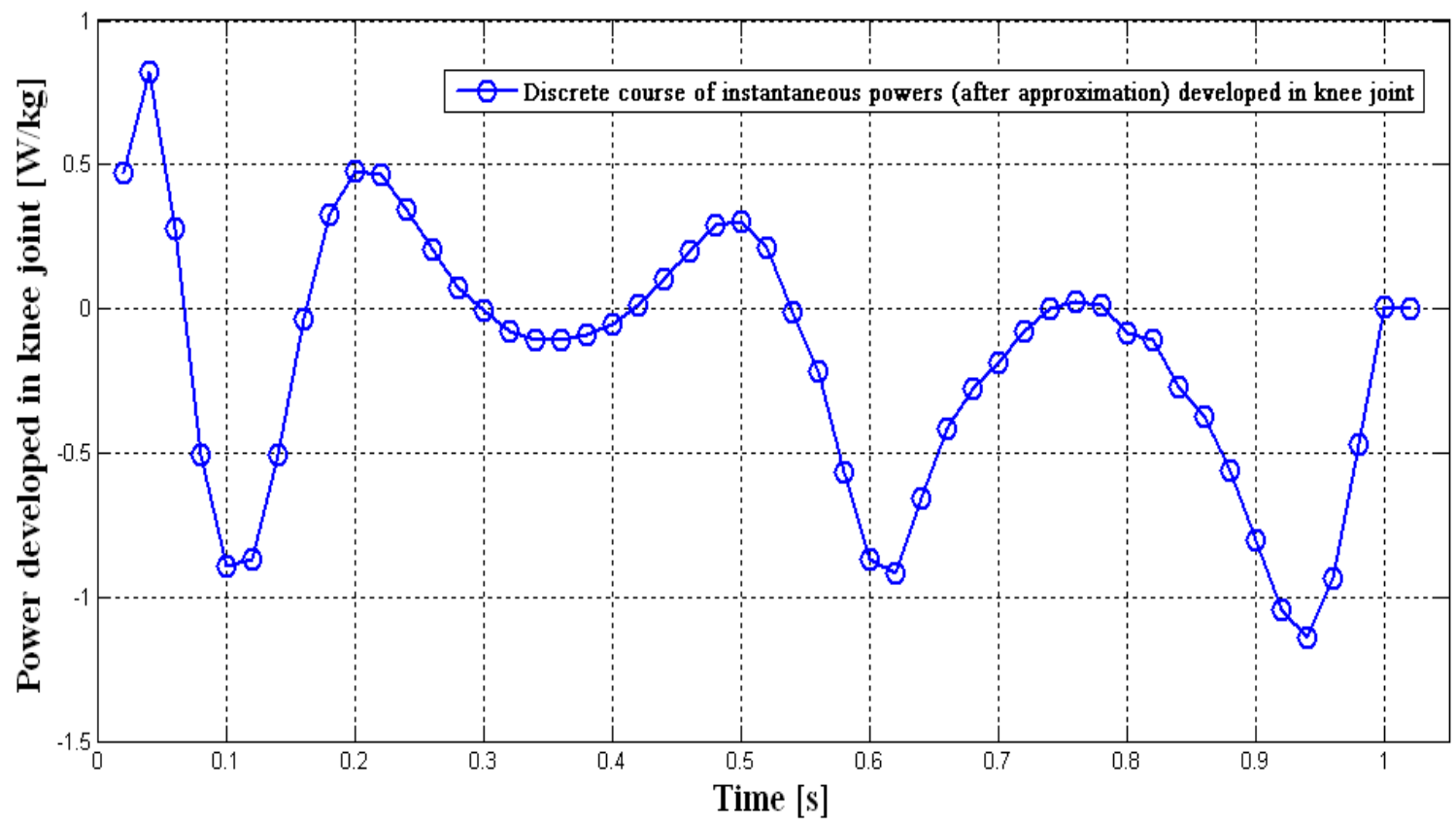

Fig.2. Discrete values of normalized powers $p_{K}(t) / m$ (after approximation) developed in knee joint of young, healthy and physically fit people (sagittal plane).

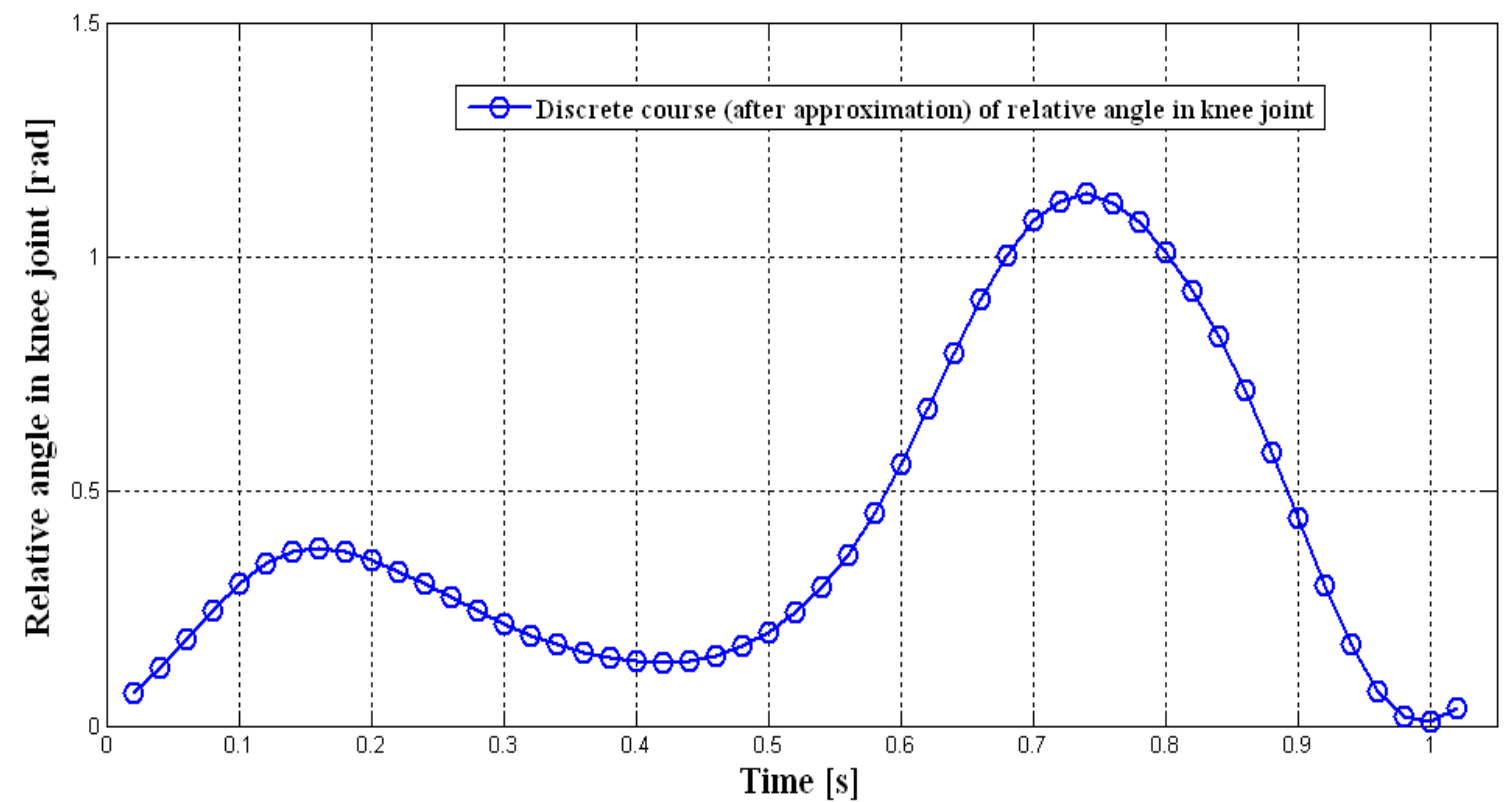

Fig.3. Discrete values of relative angle $\phi_{K}(t)$ (after approximation) in knee joint of young, healthy and physically fit people (sagittal plane). 


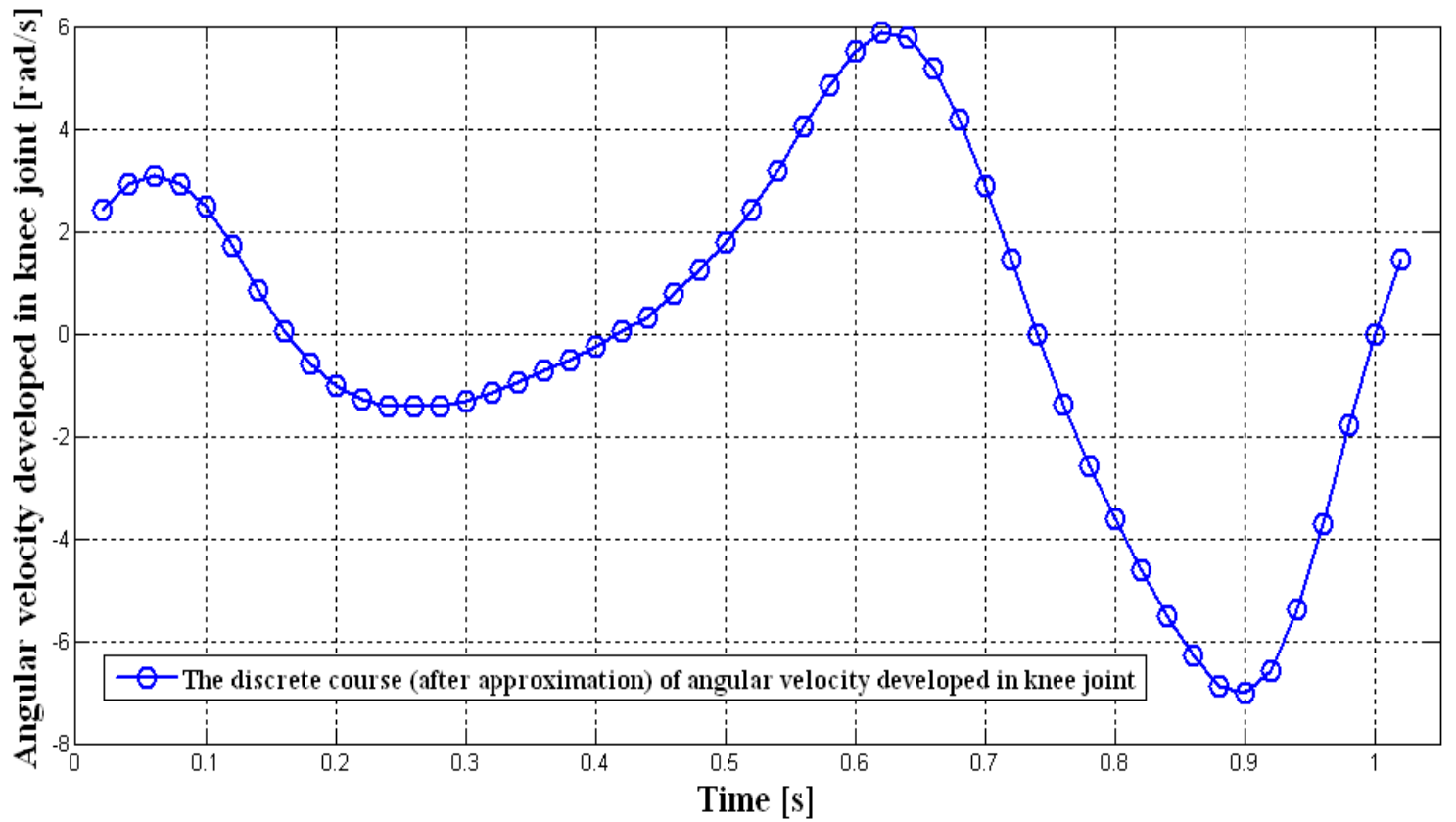

Fig.4. Discrete values of angular velocity $\dot{\phi_{K}}(t)$ (after approximation) developed in the knee joint of young, healthy and physically fit people (sagittal plane).

The angular velocity $\dot{\phi_{K}}(t)$ (Fig.4) of the knee joint was determined by numerical methods. When data such as the angular velocity $\dot{\phi_{K}}(t)$, relative angle $\phi_{K}(t)$ and power $p_{K}(t) / m$ of the knee joint are known, it is possible to find the coefficients of the state matrix [A] $a_{K}(t)$ and control matrix [B] $b_{K}(t)$.

The coefficients $a_{K}$ and $b_{K}$, which would be constant for the full cycle of movement, i.e., for one single step were calculated using identification methods (Eykhoff, 1980). Unfortunately, the attempts to find these coefficients $a_{K}$ and $b_{K}$ failed. Coefficients have a stochastic character. By integrating both sides of the equation of state (2.2) and then transfering the initial condition (2.3) to infinity, the formulas of calculating the coefficients $a_{K}$ (Fig.5) and $b_{K}$ (Fig.6) were achieved. The coefficients have the following form

$$
\begin{aligned}
& a_{K}\left(t_{i}\right)=\frac{\dot{\dot{\phi}_{K}}\left(t_{i}\right)+\frac{\operatorname{CONST}_{1}}{\operatorname{CONST}_{3}} \cdot \frac{p_{K}\left(t_{i}\right)}{m}}{\phi_{K}\left(t_{i}\right)+\frac{\mathrm{CONST}_{2}}{\mathrm{CONST}_{3}} \cdot \frac{p_{K}\left(t_{i}\right)}{m}}, \frac{1}{\mathrm{~s}}, \\
& b_{K}\left(t_{i}\right)=\frac{\mathrm{CONST}_{1}-a_{K}\left(t_{i}\right) \cdot \mathrm{CONST}_{2}}{\mathrm{CONST}_{3}}, \quad \frac{\mathrm{rad} \cdot \mathrm{kg}}{\mathrm{J}}
\end{aligned}
$$

where: $t_{i}$ - the $i$-th duration moment $(i \in\langle 1,51\rangle)$ of a full motion cycle, i.e., of one single $\left(T_{-I_{+}} \approx 1.02 s\right)$, $T_{-I_{+}}$- the duration of one step that includes both support and swing phases of the lower limb. The integral equations have the following form 


$$
\begin{array}{ll}
\int_{0}^{T_{-++}} \dot{\varphi}_{i}(t) d t=\mathrm{CONST}_{1}[\mathrm{rad}], & \int_{0}^{T_{-++}} \varphi_{i}(t) d t=\operatorname{CONST}_{2}[\mathrm{rad} \cdot \mathrm{s}], \\
\int_{0}^{T_{-++}} \frac{p_{i}(t)}{m} d t=\operatorname{CONST}_{3}\left[\frac{\mathrm{rad} \cdot \mathrm{kg}}{\mathrm{W} \cdot \mathrm{s}}\right] .
\end{array}
$$

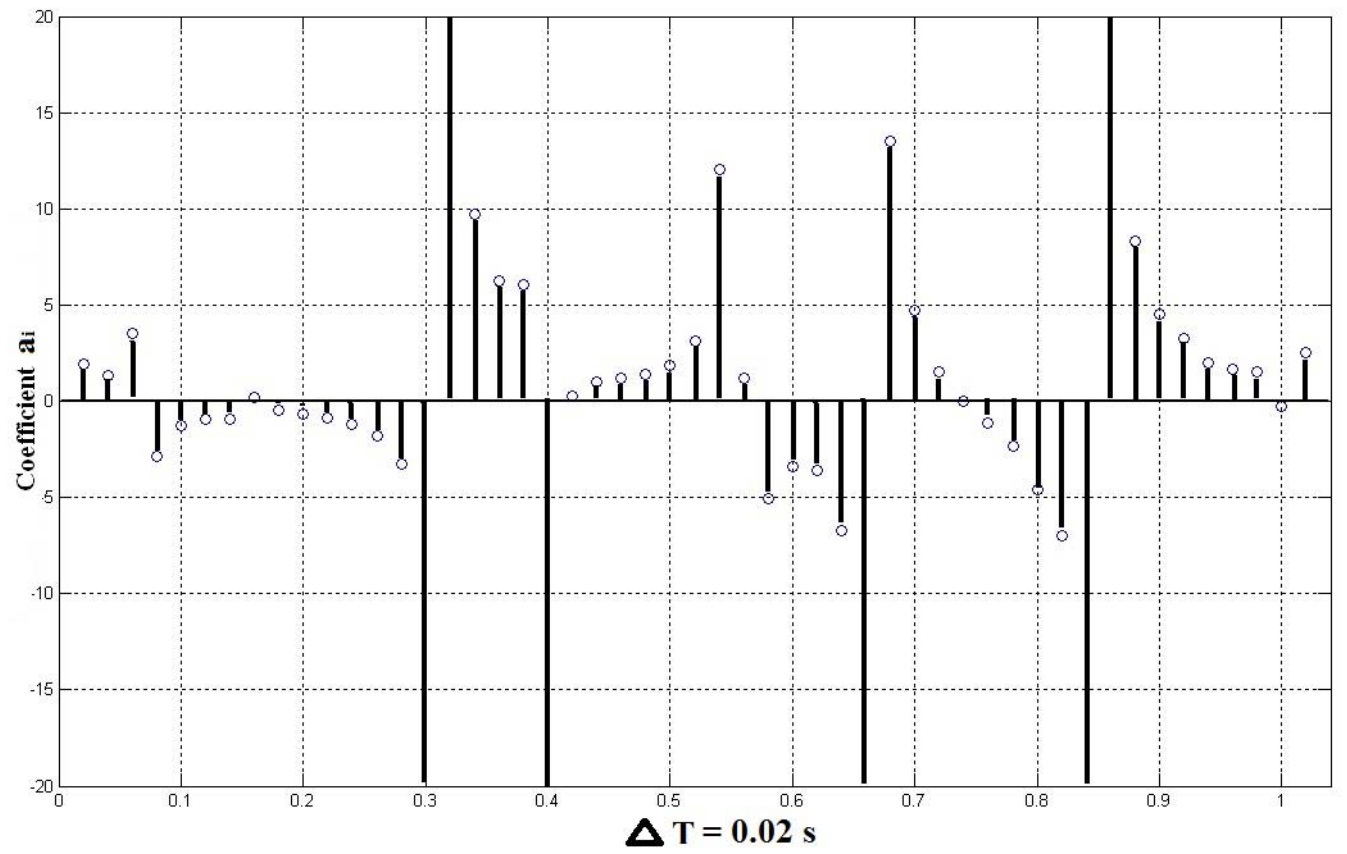

Fig.5. The values of the coefficient $a_{K}\left(t_{i}\right)$ of the knee joint of young, healthy and physically fit people.

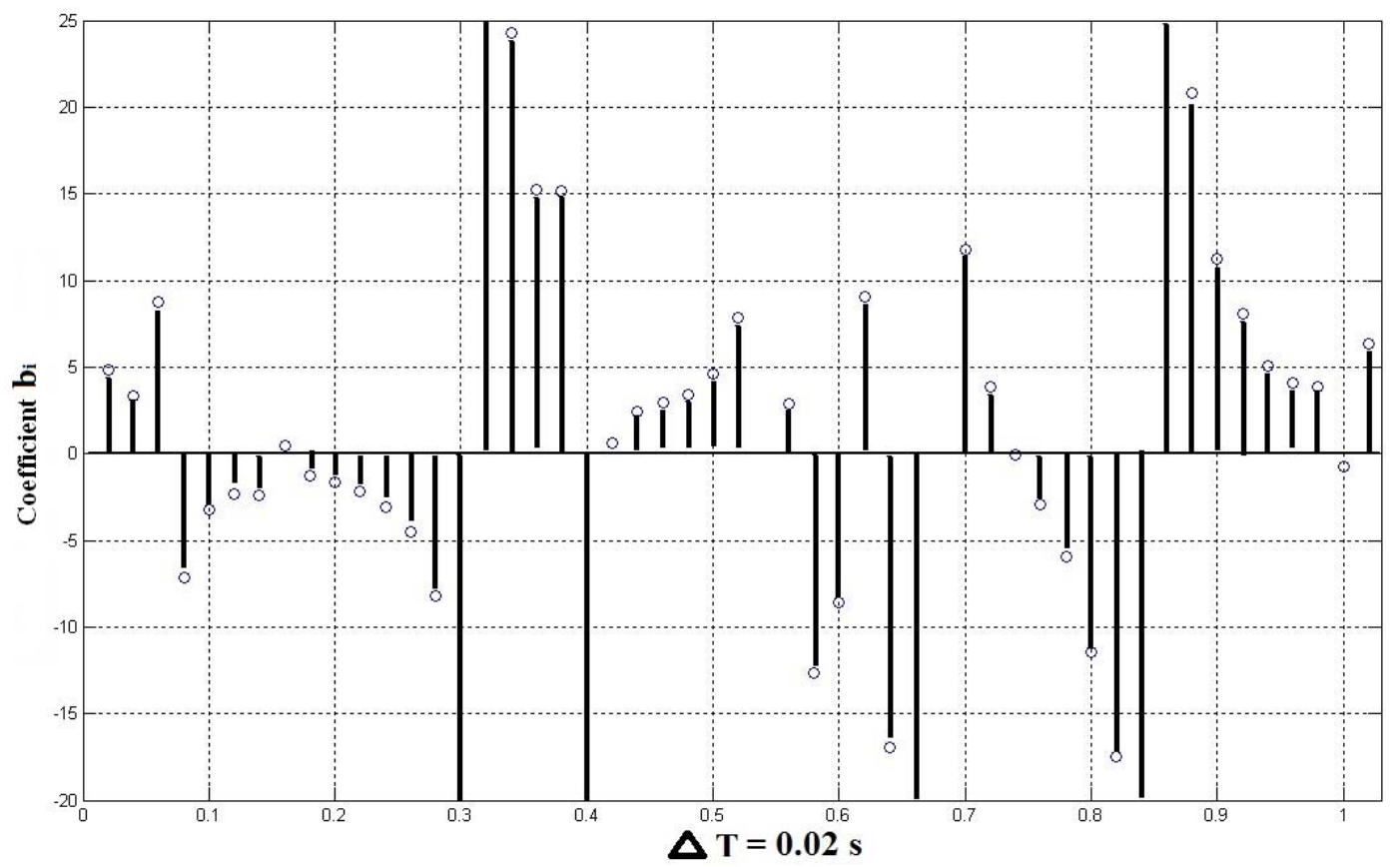

Fig.6. The values of the coefficient $b_{K}\left(t_{i}\right)$ of the knee joint of young, healthy and physically fit people. 


\subsection{The application of maximum principle for human knee joint}

A very important issue in the control theory is optimization. The maximum principle of Pontryagin is considered a good method to search the optimal conditions for controlling dynamic processes.

In the case of two-leg human locomotion, the maximum principle of Pontryagin is as follow:

if the control vector $p_{i}(t) / m$ in a given human joint is optimal, (if it minimizes the functional $J_{i}^{*}(1.3)$ ), the Hamilton function $H_{i}$ for the $i$-th joint will be maximum.

For the knee joint of young, healthy and physically fit people, equation of state (2.2) is as follows

$$
\dot{\phi_{K}}(t)=f_{K}\left(\phi_{K}(t), \frac{p_{K}(t)}{m}, t\right)
$$

with the initial condition

$$
\phi_{K}\left(t=t_{0}\right)=\phi_{K 0}
$$

For the equation of state (2.7) (on the basis of formula (1.3)) the functional has the following form

$$
J_{K}^{*}=\frac{1}{T_{-/+}} \sqrt{\int_{0}^{T_{-1+}} \frac{p_{K}^{2}(t)}{m^{2}} d t}, \quad W
$$

where: $T_{-l_{+}}$- duration of step for the support phase and the swing phase (of the lower limb) $[s], p_{K}(t) / m$ the normalized power developed by the muscle groups that operate the knee joint (Fig.2) $[\mathrm{W} / \mathrm{kg}]$.

The loss function $f_{0}$ is an integrand function of the functional $J_{K}^{*}$ Eq.(2.9), $p_{K}^{2}(t) / m^{2}$ - the square of normalized power developed by the muscle groups. For the knee joint the Hamilton function has the form (Tou, 1964)

$$
H_{K}\left(\phi_{K}(t), \frac{p_{K}(t)}{m}, \lambda_{K_{i}}, t\right)
$$

where: $\lambda_{K_{i}}$ - the conjugate variables of the state vector.

The Hamilton function $H_{K}\left(\phi_{K}(t), \frac{p_{K}(t)}{m}, \lambda_{K_{i}}, t\right)$ for the knee joint is in the following form

$$
H_{K}\left(\phi_{K}(t), \frac{p_{K}(t)}{m}, \lambda_{K_{i}}, t\right)=\lambda_{K_{0}} \cdot f_{0}+\lambda_{K_{I}} \cdot f_{K}\left(\phi_{K}(t), \frac{p_{K}(t)}{m}, t\right)
$$

and

$$
H_{K}\left(\phi_{K}(t), \frac{p_{K}(t)}{m}, \lambda_{K_{i}}, t\right)=\lambda_{K_{0}} \cdot \frac{p_{K}^{2}(t)}{m^{2}}+\lambda_{K_{I}} \cdot\left[a_{K}(t) \cdot \phi_{K}(t)+b_{K}(t) \cdot \frac{p_{K}(t)}{m}\right]
$$


The value of the first conjugate variable must be equal $\lambda_{K_{0}}=-1$ (Pontryagin, 1983). The second conjugate variable $\lambda_{K_{1}}$ was calculated from the canonical equation in the following form

$$
\begin{aligned}
& \dot{\lambda}_{K_{l}}=-\frac{\partial H_{K}\left(\phi_{K}(t), \frac{p_{K}(t)}{m}, \lambda_{K_{i}}, t\right)}{\partial \phi_{K}(t)}, \\
& \dot{\lambda}_{K_{l}}=-\lambda_{K_{l}} \cdot a_{K}(t) .
\end{aligned}
$$

The homogenous ordinary differential Eq.(2.14) was solved by the method of separation of variables (Matwiejew, 1983)

$$
\begin{aligned}
& \dot{\lambda}_{K_{1}}+\lambda_{K_{I}} \cdot a_{K}(t)=0, \\
& \lambda_{K_{1}}=C_{I} \cdot e^{-a_{K}(t) \cdot t} .
\end{aligned}
$$

The constant $C_{1}$ cannot be determined from the two-boundary problem. From the condition sine qua non of existence of optimal control (the state Eq.(2.2) is linear in the time interval $t \in\left\langle t_{i}, t_{i+1}\right\rangle$ )

$$
\begin{gathered}
\frac{\partial H_{K}\left(\phi_{K}(t), \frac{p_{K}(t)}{m}, \lambda_{K_{i}}, t\right)}{\partial \frac{p_{K}(t)}{m}}=0, \\
-\frac{2 p_{K}(t)}{m^{2}}+\frac{b_{K}(t) \cdot \lambda_{K_{I}}}{m}=0,
\end{gathered}
$$

the variable $\lambda_{K_{l}}$ has the following form

$$
\lambda_{K_{I}}=\frac{2 p_{K}(t)}{m \cdot b_{K}(t)}
$$

After calculations, the formula $C_{1}$ has the form

$$
C_{l}=\frac{2 p_{K}(t)}{m \cdot b_{K}(t) \cdot e^{-a_{k}(t) \cdot t}}
$$

The Hamilton function $H_{K}\left(\phi_{K}(t), \frac{p_{K}(t)}{m}, \lambda_{K_{i}}, t\right)$ has the form 


$$
H_{K}\left(\phi_{K}(t), \frac{p_{K}(t)}{m}, \lambda_{K_{i}}, t\right)=-\frac{p_{K}^{2}(t)}{m^{2}}+\frac{2 p_{K}(t)}{m \cdot b_{K}(t)} \cdot\left(a_{K}(t) \cdot \phi_{K}(t)+b_{K}(t) \cdot \frac{p_{K}(t)}{m}\right)
$$

After reductions, the Hamilton function has the form

$$
H_{K}\left(\phi_{K}(t), \frac{p_{K}(t)}{m}, \lambda_{K_{i}}, t\right)=-\frac{p_{K}^{2}(t)}{m^{2}}+\frac{2 p_{K}(t) \cdot a_{K}(t) \cdot \phi_{K}(t)}{m \cdot b_{K}(t)}+\frac{2 p_{K}^{2}(t)}{m^{2}} .
$$

Taking into account coefficients $a_{K}\left(t_{i}\right)(2.4)$ and $b_{K}\left(t_{i}\right)$ (2.5), discrete relative angles $\phi_{K}\left(t_{i}\right)$, normalized power $p_{K}\left(t_{i}\right) / m$ and the angular velocities $\dot{\phi}_{K}\left(t_{i}\right)$, the Hamilton function $H_{K}\left(\phi_{K}(t), \frac{p_{K}(t)}{m}, \lambda_{K_{i}}, t\right)$ may be written in the following form (Fig.7)

$$
H_{K}\left(\phi_{K}\left(t_{i}\right), \frac{p_{K}\left(t_{i}\right)}{m}, \lambda_{K_{i}}\left(t_{i}\right), t_{i}\right)=2 \frac{a_{k}\left(t_{i}\right)}{b_{k}\left(t_{i}\right)} \frac{p_{K}\left(t_{i}\right)}{m} \cdot \phi_{K}\left(t_{i}\right)+\frac{p_{K}^{2}\left(t_{i}\right)}{m^{2}},\left[W^{2} / \mathrm{kg}^{2}\right] .
$$

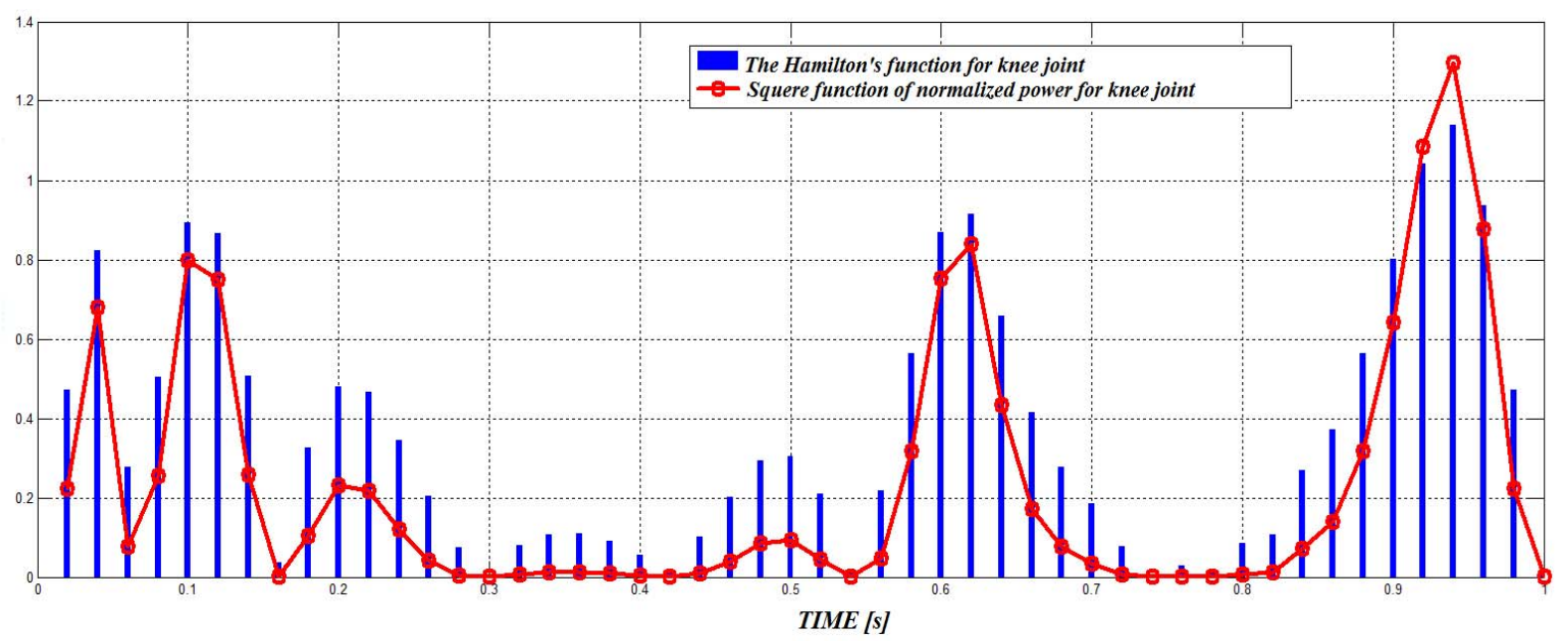

Fig.7. The discrete Hamilton function $H_{K}\left(\phi_{K}\left(t_{i}\right), \frac{p_{K}\left(t_{i}\right)}{m}, \lambda_{K_{i}}\left(t_{i}\right), t_{i}\right)$ and the square function of normalized power $p_{K}\left(t_{i}\right) / m$ developed by muscles at the knee joint

The Hamilton function $H_{K}\left(\phi_{K}\left(t_{i}\right), \frac{p_{K}\left(t_{i}\right)}{m}, \lambda_{K_{i}}\left(t_{i}\right), t_{i}\right)$ of the knee joint satisfies the optimal condition (Tou 1964)

$$
H_{K}\left(\phi_{K}\left(t_{i}\right), \frac{p_{K}\left(t_{i}\right)}{m}, \lambda_{K_{i}}\left(t_{i}\right), t_{i}\right) \geq 0
$$


The Hamilton function $H_{K}\left(\phi_{K}\left(t_{i}\right), \frac{p_{K}\left(t_{i}\right)}{m}, \lambda_{K_{i}}\left(t_{i}\right), t_{i}\right)$ for the knee human joint (Fig.7), minimizes the functional $J_{K}^{*}$ (2.9) (minimizes the energy consumption for the motion) in the full cycle of the gait (during one single step) .

\section{Conclusions}

The hypothesis about the control vector (during human motion) which is normalized power $p_{i}(t) / m$ developed by the muscle group of the $i$-th human joint makes it possible to apply the maximum principle of Pontryagin in biomechanics of human gait in the sagittal plane of motion. The above-mentioned hypothesis also enables the use of the maximum principle of Pontryagin in anthropomorphic robotics (Żur and Jaworek 2011).

The Hamiltonian for the human knee joint (Fig.7) minimizes the functional $J_{K}^{*}$ Eq.(2.9) during one single step (minimizes the energy consumption for the motion). In this way there is a possibility of shaping temporary powers $p_{i}(t) / m$ of anthropomorphic DAR type robots in order to its minimize (in the greatest number of the pulse periods $\Delta T_{i}$.) Similar research on this topic should be aimed at studying other human joints, i.e., the hip and the ankle joints. It should be continued in order to broaden specialist knowledge about the control of human and anthropomorphic robot's limbs.

\section{Nomenclature}

$$
\begin{aligned}
a_{i} & - \text { coefficients of the state matrix } \\
b_{i} & - \text { coefficients of the control matrix } \\
H_{i} & - \text { the Hamilton function } \\
J_{i}^{*} & - \text { functional of quality } \\
\overrightarrow{M_{i}}(t) & - \text { vector of moment of muscle force } \\
m & - \text { mass of the human } \\
p_{i}(t) / m & - \text { normalized power of human joints } \\
T_{-/+} & - \text {full cycle of human gait } \\
\frac{u_{i}}{\lambda_{i}} & - \text { vector of control } \\
\phi_{i} & - \text { conjugate variables } \\
\overrightarrow{\omega_{i}}(t) & - \text { vector of angular velocity }
\end{aligned}
$$

\section{References}

Bocardi S. and Pedotti A. (1981): Evaluation of muscular moments at the lower limb joints by an on-line processing of kinematics data and ground reaction. - Journal of Biomechanics, No.1, vol.14.

Eykhoff P. (1980): Identification in dynamics system. - Warsaw: PWN.

Jaworek K. (1992): Method of evaluation of human locomotion for running and walking. - Work of Institute of Biocybernetics and Biomedical Engineering Polish Academy of Sciences, vol.32, Warsaw.

Pontryagin L.S. (1983): Mathematical theory of optimal process. - Moskva: Nauka Press.

Winter D.A. (1991): The Biomechanics and Motor Control of Human Gait: Normal, Elderly and Pathological (second edition). - University of Waterloo Press. 
Tou J.T. (1964): Theory of control. - Warsaw: N-T Press.

Żur K.K. (2011): A new idea of modelling and dynamic analysis of human walking apparatus. - Acta Mechanica et Automatica, No.1, Bialystok, pp.148-153.

Żur K.K. and Jaworek K.A. (2011): New idea of control of walking anthropomorphic DAR type robot, Measurements Control Robotic - Industrial Research Institute for Automation and Measurements, No.2, Warsaw, pp.462-473.

Żur K.K. (2011): A new idea of modelling and dynamic analysis of human walking apparatus. - Acta Mechanica et Automatica, No.1, Bialystok, pp.148-153.

Żur K.K. (2013): Identification of steady and non-steady gait of human-exoskeleton walking system. - International Journal of Applied Mechanics and Engineering, vol.18, No.3, pp. 923-933.

Received: September 5, 2013

Revised: December 12, 2013 